\title{
Parametric Excitation of Vibrations in Printing Machines
}

\author{
Markus H. E. Messer \\ TH Mittelhessen (University of Applied Sciences), Fachbereich 12 (Department of Mechanical Engineering), \\ Wilhelm-Leuschner-Straße 13, 61169 Friedberg, Germany.
}

(Received 30 December 2014; accepted 4 October 2016)

In this paper the parametric excitation of vibrations in printing machines is analysed both experimentally and theoretically. Initially, selected results from experimental studies of these drive-belt-induced torsional vibrations are discussed. For this, twelve different drive belts are used during experiments at a test rig and at a sheet-fed offset printing machine. Measured vibration amplitudes from machine and test rig correlate very well. Depending on the chosen drive belt, large vibration amplitudes occur during printing in the vicinity of a critical production speed, where the first drive belt order coincides with the machine's first eigenfrequency. This results in a clearly visible rhythm during printing and is therefore unacceptable. It is demonstrated experimentally, that active vibration control can be used successfully to reduce these parametrically excited torsional vibrations in sheet-fed offset printing machines. Furthermore, the machine is modelled as a dynamical multi-degree of freedom system with time-periodic coefficients, and the parametric excitation of torsional vibrations is studied numerically. First, a numerical stability analysis is carried out employing Floquet theory. The system's damping parameters are derived from measured machine data. Accordingly, a simulation model with drive control results in parametrically excited torsional vibration amplitudes that match very well with measured amplitudes.

\section{NOMENCLATURE}

$\alpha \quad$ mass-proportional damping parameter

$\beta \quad$ stiffness-proportional damping parameter

$c \quad$ drive belt stiffness

$D \quad$ modal damping (damping ratio)

$\underline{D}_{0} \quad$ damping matrix

dof degrees of freedom, degree of freedom

$d_{s} \quad$ speed control parameter

$\varepsilon \quad$ small quantity

$f \quad$ drive torque

$f$ torque vector

FFT fast Fourier transform

$\underline{K}_{0} \quad$ stiffness matrix

$k_{p} \quad$ position control parameter

LTI linear time-invariant

LTP linear time-periodic

$\underline{M}_{0} \quad$ mass matrix

$P E$ parametric excitation

$q \quad$ angular displacement vector

$\bar{q}_{\text {ref }}$ reference angular position

$\dot{q} \quad$ angular velocity vector

$\overline{\dot{q}}_{\text {ref }}$ reference angular velocity

$\ddot{q} \quad$ angular acceleration vector

$R^{2} \quad$ coefficient of determination

sph sheets per hour

$t$ time

$T$ time period

$v \quad$ production speed

$\underline{\varphi}_{1} \quad$ first eigenform (mode shape)

$\omega_{P E} \quad$ parametric excitation frequency

$\omega_{1} \quad$ first eigenfrequency

\section{INTRODUCTION}

Figure 1 schematically shows a printing machine, whose torsional vibrations are studied in this paper. Numbers 1 to 19 correspond to the rotational dof in the dynamical system that will be explained later in detail. The machine illustrated in Fig. 1 is a so-called Speedmaster XL 162-6+L, which represents a typical sheet-fed offset printing machine produced by Heidelberger Druckmaschinen AG. This type of machine has a length of $25.9 \mathrm{~m}$ and a height of $3.4 \mathrm{~m}$, and it consists of feeder and delivery unit as well as six printing units and a coating unit as illustrated in Fig. 1. The maximum sheet size that can be handled by this machine is equal to $1.62 \mathrm{~m} \times 1.21 \mathrm{~m}$.

Currently, fastest sheet-fed offset printing machines print 18,000 sheets per hour $(5 \mathrm{~Hz})$, and typical print products of high quality include magazines, catalogues, brochures, business cards, posters, flyers, books, packages, wrappers, etc. All cylinders in a sheet-fed offset printing machine are coupled by gear wheels. Consequently, one single main drive is used to drive the whole machine via a gear train. The drive torque is transmitted by one V-ripped drive belt. This single drive belt leads to the parametric stiffness excitation of vibrations examined in this paper. In Fig. 1, the small belt pulley (dof 19) is driven by a main drive. The drive belt then transmits the drive torque to the large belt pulley (dof 18) that drives the impression cylinder of printing unit \#1 (dof 2) etc.

Dof 1-14 are so-called paper-transporting cylinders. These cylinders contain gripper systems for transporting the sheets of paper. Each printing unit operates on three main cylinders: plate cylinder, blanket cylinder, and impression cylinder (see Fig. 1). Printing units also contain inking and dampening unit. During printing, these units contact the plate cylinder to cover the printing plate with a thin film of ink and dampening so- 


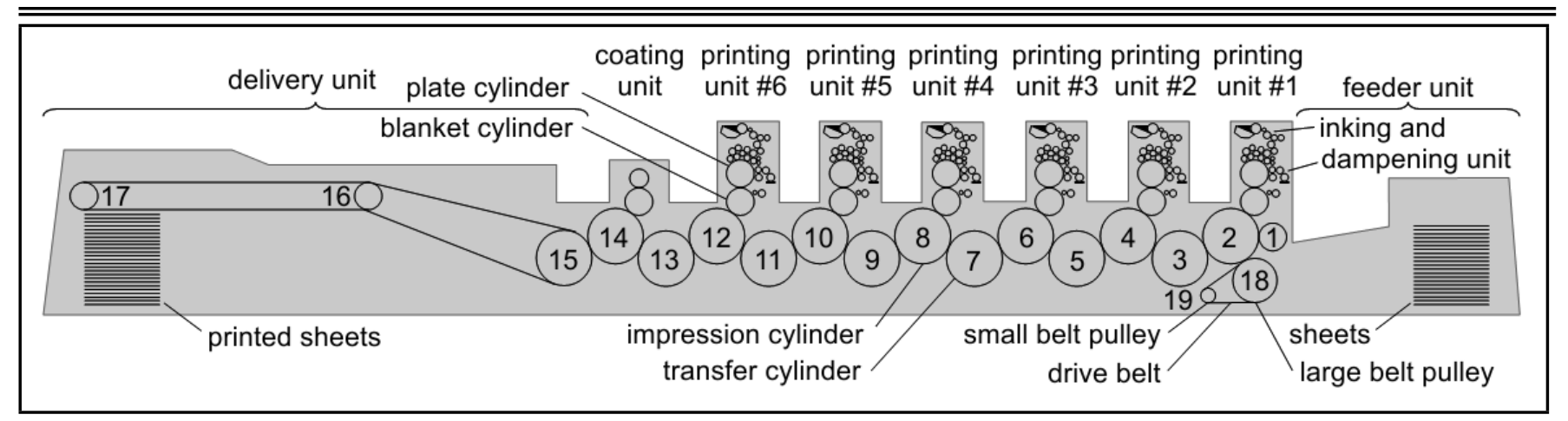

Figure 1. Sheet-fed offset printing machine (Speedmaster XL 162-6+L, Heidelberger Druckmaschinen AG).

lution (water plus additives). The plate cylinder is in contact with the blanket cylinder. Next, the blanket cylinder contacts the impression cylinder, which holds the sheet of paper being printed with grippers. Further information concerning printing machines and technologies can be obtained from the extensive textbooks by Kipphan (Ed.). On more than 1200 pages, these books contain numerous details. ${ }^{1,2}$

In general, vibrations in printing machines have a negative effect on print quality and often hinder an increase in productivity. Printed dot sizes of $20 \mu \mathrm{m}$ are typical in sheet-fed offset printing, and cylinder vibrations with an amplitude of only $5 \mu \mathrm{m}$ can be visible in print. ${ }^{3}$

The first known dynamic analysis of sheet-fed offset printing machines was conducted by Holzweißig, who examines gear-induced torsional and bending vibrations of a sheet-fed offset printing machine. ${ }^{4}$ Yamaguchi et al. present a vibration absorber to reduce gap-induced bending vibrations. ${ }^{5}$ Buck compares simulated and measured torsional vibrations in sheet-fed offset printing machines and gives modelling details. ${ }^{6}$ Compensation mechanisms and active vibration control are used effectively against torsional vibrations. ${ }^{7}$ Buck et al. present non-linear torsional vibration phenomena in sheet-fed offset printing machines. ${ }^{8}$

The machine in Fig. 1 uses a V-ripped L-profile drive belt, whose total thickness is $10 \mathrm{~mm}$ (DIN 7867). ${ }^{9}$ The drive belt consists mainly of vulcanized rubber material and contains a load-carrying tension member, i.e. high strength fibres that transmit the longitudinal forces. The diameter, where the longitudinal forces are acting, is referred to as effective diameter. Dresig examines the differential equation of parametrically excited Euler-Bernoulli beam vibrations and gives an equation for instability boundary curves of parametrically excited drive belt vibrations. ${ }^{10}$ Dresig considers eccentricity of one belt pulley that leads to a harmonic variation of the longitudinal drive belt forces and results in a harmonic parametric stiffness excitation. ${ }^{10}$ Sauer analyses stationary vibrations of a drive belt system and presents dynamical models for parametrically excited drive belt vibrations. ${ }^{11}$ Sauer points out that drive belt width and thickness are not constant along the belt's length. ${ }^{11}$ Accordingly, these inhomogeneities result in a periodic variation of the effective diameter of the pulleys. The period of that variation corresponds to one belt cycle. Changes of the effective diameter of the pulleys lead to an alteration of longitudinal drive belt forces, causing parametrically excited drive belt vibrations. Bischoff demonstrates that the aforementioned changes of the effective diameter of the pulleys can be mod-

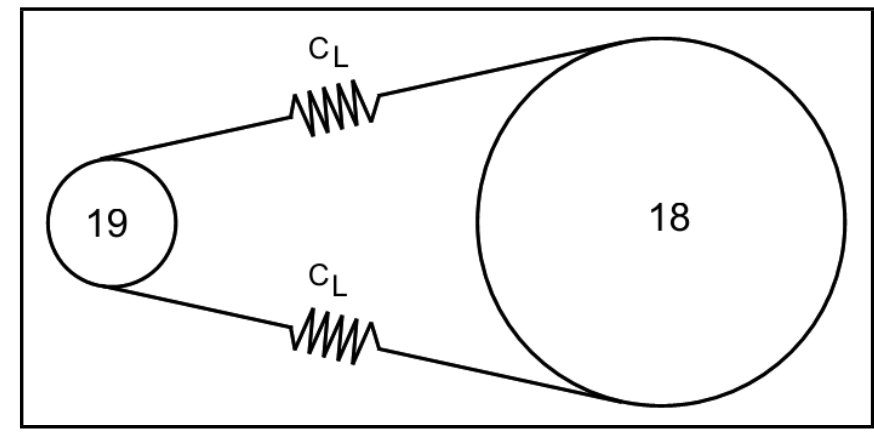

Figure 2. Drive belt model.

elled as a variation of the drive belt stiffness, leading to parametrically excited torsional vibrations in sheet-fed offset printing machines. ${ }^{12}$

In accordance with the argumentations of Dresig, ${ }^{10}$ Sauer $^{11}$ and Bischoff, ${ }^{12}$ the drive belt is therefore modelled as a spring whose stiffness is not constant. This is equivalent to a so-called parametric stiffness excitation. Figure 2 illustrates the drive belt model used in this work. Here, $c_{L}$ represents the drive belt's longitudinal stiffness that couples small and large belt pulley (dof 19 and 18, see Fig. 1).

The present paper offers the following new contributions:

a) Results from experimental studies, e.g. experiments at a test rig and at a sheet-fed offset printing machine. Measured vibration amplitudes correlate very well.

b) Experimental demonstration of success in using active vibration control to reduce parametrically excited torsional vibrations in sheet-fed offset printing machines.

c) Modelling of the machine as a dynamical multi-degree of freedom system with time-periodic coefficients (numerical stability analysis employing Floquet theory; dynamical model with drive control; and damping parameters from measurement data).

d) Comparison of simulated and measured parametrically excited torsional vibrations in sheet-fed offset printing machines. Numerical and experimental results match very well.

\section{MEASUREMENTS OF PARAMETRICALLY EXCITED TORSIONAL VIBRATIONS}

Parametrically excited torsional vibrations are a typical problem in sheet-fed offset printing machines. Figure 3 a shows 


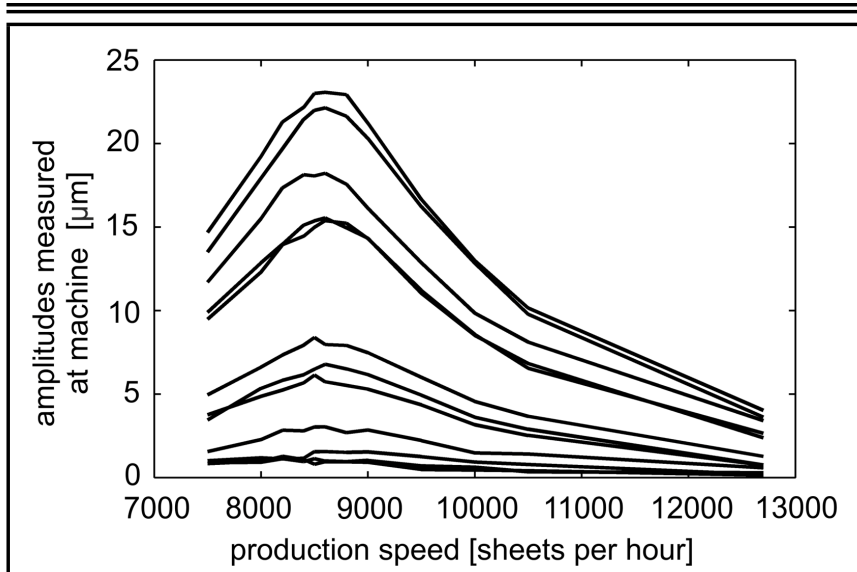

(a)

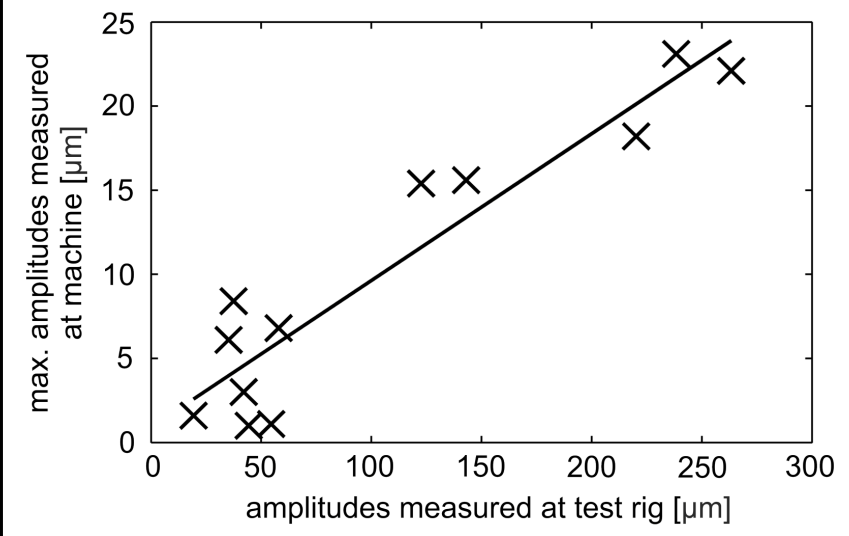

(b)

Figure 3. (a) Torsional vibration amplitudes of frequency $\omega_{P E}$ measured at machine, (b) maximum amplitudes measured at machine versus amplitudes measured at test rig.

amplitudes of frequency $\omega_{P E}$ (drive belt's rotation frequency). For these experiments twelve different drive belts were used at a Speedmaster XL 162-6+L, and near a critical production speed $(8,600$ sheets per hour) torsional vibrations were measured with rotary shaft encoders. All these twelve drive belts were of the same type and from the same manufacturer. The drive belt completes 3.24 periods while one sheet of paper is being printed. Hence, the highest parametric excitation frequency could be reached at a maximum production speed of 18,000 sheets per hour and is equal to $16.2 \mathrm{~Hz}(3.24 \cdot 5 \mathrm{~Hz})$. Large vibration amplitudes occur near 8,600 sheets per hour, where $\omega_{P E}$ coincides with the machine's first eigenfrequency $\omega_{1}(7.7 \mathrm{~Hz})$. In that case some drive belts lead to amplitudes greater than $20 \mu \mathrm{m}$. This results in a clearly visible rhythm during printing and is for that reason absolutely intolerable.

The twelve drive belts, which were used for the experiments illustrated in Fig. 3a, were all tested on a test rig that consisted of two belt pulleys and a rotary shaft encoder. At the test rig, torsional vibration amplitudes were measured for each drive belt. Figure $3 \mathrm{~b}$ is obtained by plotting maximum amplitudes measured at the machine versus amplitudes determined at the test rig. The data depicted in Fig. 3b exhibit significant correlation. Employing linear regression yields a coefficient of determination of $R^{2}=0.88$. Consequently, the selection of drive belts, based on measured torsional vibration amplitudes at a test rig, is one possibility to handle the problem of these drive-belt-induced torsional vibrations. By measuring all drive

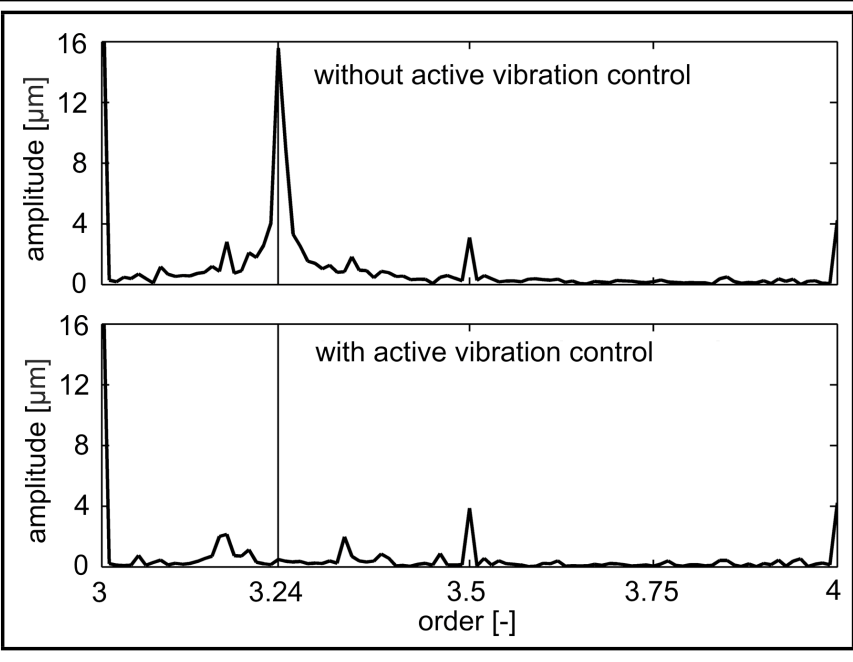

Figure 4. Amplitudes of torsional difference vibration (dof $10-$ dof 2) measured at machine.

belts on a test rig and allowing only selected drive belts to be installed into the machines, it is therefore possible to maintain the amplitudes during printing below a critical value.

Active vibration control is another possibility to reduce these drive-belt-induced torsional vibrations. Figure 4 shows torsional vibration amplitudes that were measured at the machine at critical production speed $\left(\omega_{P E}=\omega_{1}\right)$ with one of the worst drive belts from Fig. 3. The amplitudes are plotted over an order axis and drive belt order 3.24 is marked, which corresponds to the parametric excitation frequency $\omega_{P E}$.

It is necessary to point out, that non-integer orders, such as order 3.24, are always unwanted in sheet-fed offset printing, because they disturb the sheet transport between neighbouring cylinders differently for consecutive sheets. Depending on the amplitude, this can lead to a noticeable rhythm in a series of printed sheets. Integer orders, such as order 3 , order 4 , etc., cause no rhythm in print. Therefore, integer orders are not a problem, as long as they do not lead to backlash and non-linear torsional vibration phenomena in the gear train, which is not the case here.

The upper part of Fig. 4 illustrates the typical problem of drive-belt-induced torsional vibrations, where at critical production speed the measured amplitude of torsional difference vibration (dof $10-\operatorname{dof} 2$ ) of order 3.24 is relatively large $(16 \mu \mathrm{m})$, which is unacceptable. In the lower part of Fig. 4 , the parametrically excited vibration amplitude of the non-integer order 3.24 is smaller than $1 \mu \mathrm{m}$ even though the same drive belt is used. This vibration reduction is achieved with active vibration control. For the active vibration control, the existing main drive of the machine is used as an actuator to counter fight unwanted vibration as described in Nöll's work that is accessible via the internet. ${ }^{13}$ The controller, which led to the results shown in Fig. 4, employs the so-called internal model principle. Of course, other control algorithms, such as adaptive harmonic control, can also be used successfully. By measuring the machine's transfer function (see Fig. 9a), most of the control parameters can be obtained (i.e., resonance frequency, amplitude, and phase angle of transfer function at resonance frequency). The only remaining control parameter needed for the internal model principle is a gain factor (see chapter 4.2 in Nöll's work for more details and recommendations regarding 
this gain factor). ${ }^{13}$

Figure 4 demonstrates that active vibration control is another possibility to handle the problem of drive-belt-induced torsional vibrations. By using active vibration control, it is not necessary to measure drive belts on a test rig and install only selected drive belts into the machine.

At first glance, the problem described in this section looks like a classical forced vibration resonance problem, but measured vibration data from different machine types (plate cylinder diameters ranging from $0.180 \mathrm{~m}$ to $0.4854 \mathrm{~m}$ ) seem to exhibit characteristics of parametrically excited vibrations, e.g. problem occurs in wide ranges of production speed. Often, amplitudes of multiple drive belt orders are visible. In order to further explore the underlying mechanism of the parametric excitation and gain a better understanding of the problem presented above, a dynamical system with time-periodic coefficients is studied in this work.

\section{DYNAMICAL SYSTEM}

In many cases, the torsional vibration behaviour of a sheetfed offset printing machine can be described by an LTI-system with proportional damping (eq. (1)). Comparisons of measurements and LTI-simulations were carried out for different torsional vibration excitations. ${ }^{3,6,7}$

$$
\underline{M}_{0} \underline{\ddot{q}}+\underline{D}_{0} \underline{\dot{q}}+\underline{K}_{0} \underline{q}=\underline{f} \text { with } \underline{D}_{0}=\alpha \underline{M}_{0}+\beta \underline{K}_{0} .
$$

The printing machine shown in Fig. 1 is modelled as a dynamical system with 19 rotational dof. Of course, one can model this type of machine with more or less degrees of freedom, depending on the vibration problem. For example, if relative vibrations between plate and blanket cylinder or blanket and impression cylinder are of interest, the model has to have dof for plate and blanket cylinder. This is only necessary, if higher eigenfrequencies are excited. The comparisons of measured and simulated vibration in this article will show, that 19 dof are more than enough. This is not surprising, since this type of model with the same dof has been used by other authors as well, and measured and simulated results matched very well in many cases. , $^{3,7}$

Figure 1 shows all 19 dof. Dof 1-14 represent paper transporting cylinders, adjacent dof 15,16 , and 17 represent the delivery unit, whereas dof 18 and 19 stand for the belt pulleys of the main drive unit. Dof 2 and 18 are coupled. The drive belt stiffness $c$ couples dof 18 and 19. Consequently, the following elements of the 19-by-19 stiffness matrix exhibit the drive belt stiffness parameter: $(19,19)$ (positive value), $(18,19)$ and $(19$, 18) (both negative). In addition to that, the drive belt stiffness contributes to element $(18,18)$ of the stiffness matrix. Therefore, in the case of a time-periodic drive belt stiffness $c$, the following four elements of stiffness and (proportional) damping matrix are time-periodic: $(18,18),(19,18),(18,19)$ and $(19,19)$.

Apart from elements $(18,2)$ and $(2,18)$, the stiffness matrix exhibits a diagonal band structure, as can be expected from Fig. 1. The mass matrix is a diagonal matrix containing the mass moments of inertia.

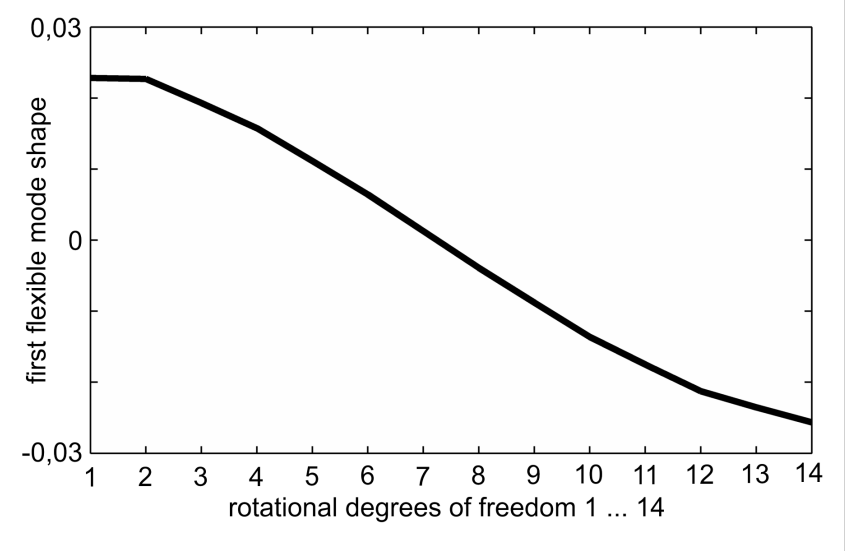

Figure 5. First flexible mode shape $\underline{\varphi}_{1}$.

Since the structure is an unconstrained system, there exists a rigid body mode shape, which is of no interest to this vibration analysis. Figure 5 shows the first flexible mode shape $\underline{\varphi}_{1}$ that belongs to the first eigenfrequency $\omega_{1}$. For easier visualization, only rotational dof 1-14 are plotted in Fig. 5. The node of this mode shape appears somewhere in the middle of the structure, and maximum values of this mode shape appear near the ends, as is typical for unconstrained systems.

Since dof 2 and 18 are coupled, the main drive unit (drive belt) can excite the first flexible mode shape very well. On the other hand, the existing main drive of the machine can be used effectively as an actuator for active vibration control, as could be seen in Fig. 4. Rotational dof 2 and 12 (or 10) represent appropriate sensor locations for rotary shaft encoders, because the first flexible mode shape exhibits sufficiently large values at these dof.

\section{NUMERICAL STABILITY ANALYSIS}

As mentioned previously, drive belt inhomogeneities result in a periodic variation of the drive belt stiffness. In the following, a harmonic variation of the drive belt stiffness is considered so that the drive belt stiffness oscillates between $c \cdot(1+\varepsilon)$ and $c \cdot(1-\varepsilon)$. The damping parameters $\alpha$ and $\beta$ are chosen so that the modal damping is fairly small $\left(D_{1}=D_{2}=0.005\right)$ for $\varepsilon=0$ (LTI). Accordingly, the following system of coupled linear differential equations with periodic (harmonic) coefficients allows the study of parametric excitation. Index 0 denotes constant matrices, index c marks matrices that are multiplied with time-periodic terms for $\varepsilon>0$.

$$
\begin{aligned}
\underline{M}_{0} \underline{\ddot{q}}+\left(\alpha \underline{M}_{0}+\beta \underline{K}_{0}+\beta \varepsilon \underline{K}_{c} c \cos \left(\omega_{P E} t\right)\right) \underline{\dot{q}} \\
+\left(\underline{K}_{0}+\varepsilon \underline{K}_{c} c \cos \left(\omega_{P E} t\right)\right) \underline{q}=\underline{0} .
\end{aligned}
$$

Equation (2) is used for stability analysis and contains no external forcing. This equation can be written as a system of first order differential equations (state space) with time-varying coefficients having time period $T_{P E}$. Employing fourth-order Runge-Kutta method, ${ }^{14}$ numerical results are obtained for independent sets of initial conditions. The use of modal coordinates allows the verification of numerical results by comparing numerical and analytical solutions for $\varepsilon=0$. Floquet theory 


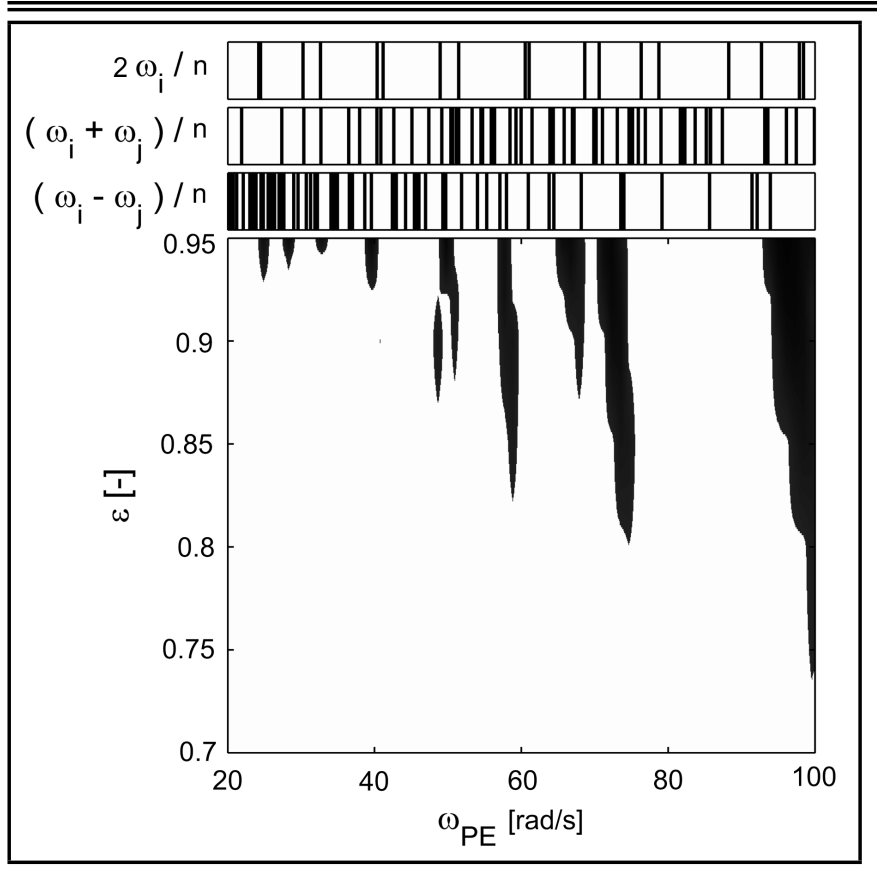

Figure 6. Instability regions (shaded), parametric resonance frequencies ( $n=$ $1,2, \ldots, 5)$.

is employed, and the eigenvalues of the monodromy matrix at time $t=T_{P E}$ are calculated in order to identify instability regions. ${ }^{15}$ Figure 6 shows selected numerical results of the stability analysis. The simulated time-periodic system exhibits instability regions (see the shaded areas in Fig. 6). The style of visualization utilized in Fig. 6, i.e., instability regions and parametric resonance frequencies, was first used by Dohnal. ${ }^{16}$

One has to keep in mind, that in this exemplary simulation the modal damping is rather small $\left(D_{1}=D_{2}=0.005\right)$. At the same time the parameter $\varepsilon$ has to be rather large $(\varepsilon>0.7)$ for the system to become instable. In the following section, measurement data from a sheet-fed offset printing machine shown in Fig. 1 are used to determine realistic damping parameters of the dynamical system. In addition to that, the drive control of the machine is modelled and included into the dynamical system. It is necessary to simulate a dynamical system with drive control, because the drive control leads to a drive torque which is generated by the main drive and is being transmitted by the drive belt. Furthermore, the drive control contributes significantly to the damping of the system, as can be seen in the following section. Thus, a realistic drive torque in combination with a time-periodic drive belt stiffness should result in simulated drive-belt-induced torsional vibrations similar to the measured vibration data shown in Fig. 3a.

\section{DRIVE CONTROL AND DAMPING}

The following Fig. 7 schematically illustrates the drive control of the sheet-fed offset printing machine. A machine operator sets a desired production speed $\dot{q}_{\text {ref }}$ which then acts as a reference speed (angular velocity) used for the drive control. The difference of this reference angular velocity and the actual angular velocity of dof 19 (main drive) is multiplied by the speed control parameter $d_{s}$. The result is the torque $f_{s}$. Since $d_{s}$ multiplies angular velocity, the speed control parameter $d_{s}$ influences the damping of the dynamical system. This

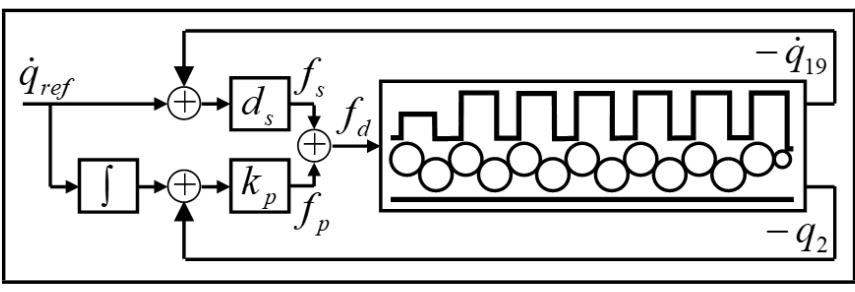

Figure 7. Drive control of the sheet-fed offset printing machine

will be analysed further below. The drive control consists not only of speed control, but contains also a position control. For position control, the reference angular velocity is integrated, which leads to a reference angular position $q_{r e f}$. The difference of this reference angular position and the actual angular position of dof 2 is multiplied by the position control parameter $k_{p}$. This results in the torque $f_{p}$. The drive torque $f_{d}$ is the sum of speed and position control torques. Accordingly, the main drive generates the drive torque $f_{d}=f_{s}+f_{p}$ which is then being transmitted by the drive belt.

Figure 8 a shows measured machine data, i.e., desired production speed (grey line) and measured drive torque (black line). At time $t=0$, the machine is standing still. The machine operator sets different desired production speeds $\left(v_{1}, v_{2}\right.$, $\left.v_{3}\right)$. Consequently, the machine accelerates towards and then runs stationary at these distinct production speeds $\left(v_{1}\right.$ : time span $6 \mathrm{~s}-9 \mathrm{~s}, v_{2}$ : time span $11 \mathrm{~s}-21 \mathrm{~s}, v_{3}$ : time span $39 \mathrm{~s}-$ $50 \mathrm{~s})$. Between these different speed levels $\left(0, v_{1}, v_{2}, v_{3}\right)$, the desired production speed is increased linearly with the same slope during each acceleration period. While the machine is accelerating towards velocity $v_{3}$, the drive torque reaches its maximum allowed value. As a result, the drive torque is a flat line between $32 \mathrm{~s}$ and $39 \mathrm{~s}$.

Taking a closer look at the data at constant velocity (stationary speeds $\left.v_{1}, v_{2}, v_{3}\right)$ and plotting the corresponding stationary drive torques (mean values) over the stationary speeds leads to Fig. 8b. There is a perfectly linear relationship between stationary drive torques and stationary speeds. As a result, the model must contain a constant torque and a linearly increasing torque. This linearly increasing torque can be modelled with the mass-proportional damping parameter $\alpha$. Choosing $\alpha$ properly, the mass proportional part of the damping matrix results in a torque that is linearly increasing with velocity as pictured in Fig. 8b.

The speed control parameter $d_{s}$ influences the damping of the dynamical system. Figure 9a shows measured transfer functions (black lines) for three different values of the speed control parameter $d_{s}$ (nominal value, $75 \%$ of the nominal value and $50 \%$ of the nominal value). The measured transfer functions were obtained by using the main drive as an actuator to excite the structure harmonically at different frequencies. For Fig. 9a, the resulting torsional difference vibrations (dof 10 - dof 2) were measured. Figure 9a illustrates the magnitude of the transfer function, i.e., the ratio of the torsional vibration amplitude (dof 10 - dof 2) and the excitation amplitude (main drive). Maximum magnitudes of the transfer function are measured at the first eigenfrequency, whose mode shape can be seen in Fig. 5. In addition to the measured transfer functions, Fig. 9a contains simulated transfer functions (LTI-system) for different modal damping values $D_{1}$. 


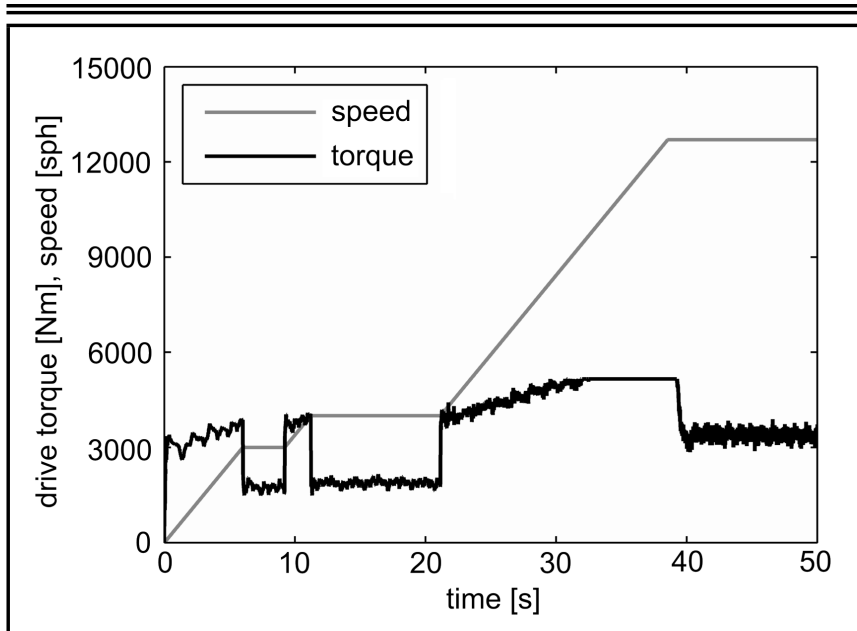

(a)

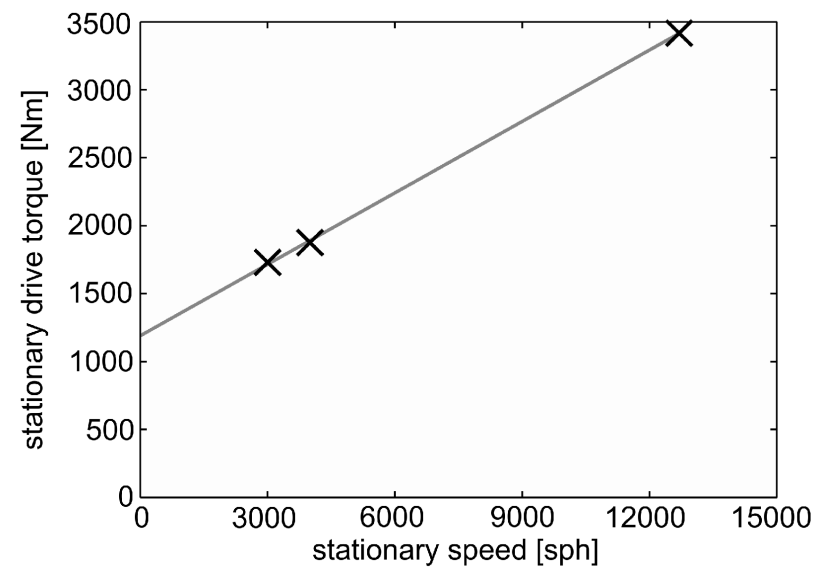

(b)

Figure 8. (a) Measured machine data: drive torque (black) and desired speed (grey), (b) stationary drive torques (mean values from measured data) over speed.

For each of the three measured transfer functions in Fig. 9a, one can determine the best matching modal damping value. In Fig. 9b, the resulting modal damping values 0.0753 , 0.0897, 0.1033 are plotted over the corresponding speed control parameter values $\left(0.5 \cdot d_{s}, 0.75 \cdot d_{s}, d_{s}\right)$. The result is a perfectly linear relationship. The highest modal damping value (0.1033) is more than factor 20 larger than the modal damping used for the stability analysis presented in the previous section. Extrapolating the linear function shown in Fig. $9 \mathrm{~b}$ leads to the modal damping value $D_{1}=0.0475$ for the dynamical system without speed control. With this value $(0.0475)$ and $\alpha$, one can now determine the structure's stiffness-proportional damping parameter $\beta$ by using Eq. (3), which can be found in any standard dynamics textbook.

$$
D_{1}=0.0475=\frac{\alpha}{2 \omega_{1}}+\frac{\beta \omega_{1}}{2} \Leftrightarrow \beta=\frac{2 \cdot 0.0475}{\omega_{1}}-\frac{\alpha}{\omega_{1}^{2}} .
$$

With parameters $\alpha$ and $\beta$ and a model of the drive control, one can simulate realistic drive torques. The simulated torque $f_{d}$ is also limited to the maximum value (flat line) of the measured torque that can be seen in Fig. 8a. In order to check the simulation model, the desired production speed from Fig. 8a is used for a simulation (LTI). Figure 10 shows the resulting drive torque.

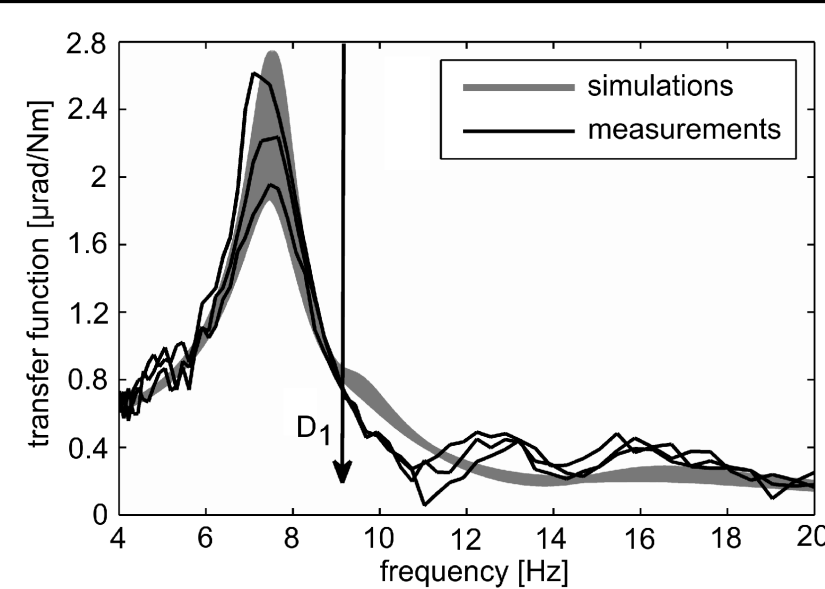

(a)

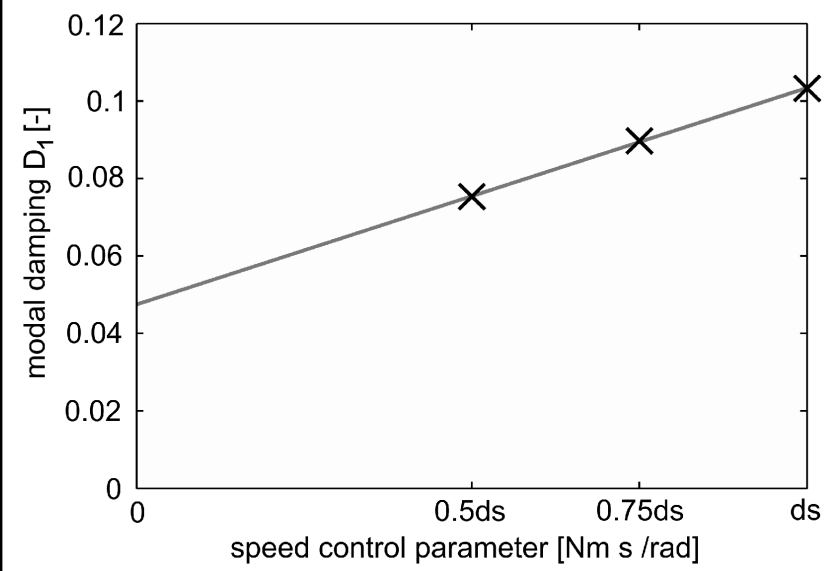

(b)

Figure 9. (a) Simulated (LTI) and measured transfer functions, (b) modal damping over speed control parameter.

The black line in Fig. 10 represents the measured torque from Fig. 8a, whereas the grey line is simulated (LTI). Measured and simulated torques do not match perfectly. Especially the transient behaviour looks quite different. Of course, the measured signal is influenced by many different excitations (e.g. opening and closing of gripper systems, unbalances of cylinders, cylinder gap shocks, vibrator rollers, etc.), ${ }^{7}$ which are not considered in this simulation. Nevertheless, measured and simulated stationary drive torques match very well at stationary speeds $\left(v_{1}, v_{2}, v_{3}\right)$.

\section{SIMULATION OF PARAMETRICALLY EXCITED TORSIONAL VIBRATIONS}

Comparing measured and simulated stationary drive torques leads to the conclusion that the simulation model can be used to study stationary (steady state) drive-belt-induced torsional vibrations, i.e., parametrically excited torsional vibrations. In order to do so, the LTI-system is changed to an LTP-system by considering a harmonic variation of the drive belt stiffness as described above. To give an example, Fig. 11 shows results of an LTP-simulation. In this simulation, the machine is accelerated towards and then run stationary at a production speed $v$ (see grey line in Fig. 11a). The black line in Fig. 11a represents the corresponding drive torque. As expected for the LTPsystem, the drive torque exhibits oscillations even at a station- 


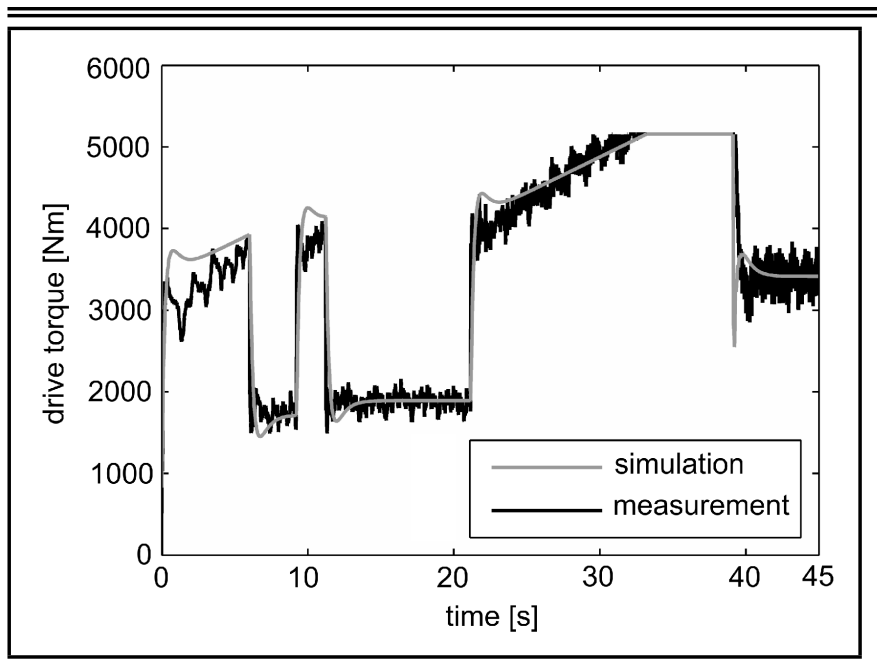

Figure 10. Simulated (LTI) and measured drive torque.

ary speed $v$, because of the time-periodic drive belt stiffness. This leads to torsional vibrations of the cylinders. The corresponding torsional difference vibration (dof $12-\operatorname{dof} 2$ ) is shown in Fig. 11b. The last part of that signal (e.g. time span $24 \mathrm{~s} \ldots 25 \mathrm{~s}$ ) is a simple harmonic function oscillating with parametric excitation frequency $\omega_{P E}$ (order 3.24) and having a typical amplitude of $13.5 \mu \mathrm{m}$.

As mentioned above, torsional vibration amplitudes of frequency $\omega_{P E}$, that were measured at a machine, exhibit large values at a critical production speed $v_{\text {crit }}$, where $\omega_{P E}=\omega_{1}$ (first eigenfrequency). Therefore, LTP-simulations are done for production speeds $v$ in the vicinity of $v_{\text {crit }}$. These simulations are carried out for the following values of $\varepsilon 0.025,0.05$, $0.075,0.1,0.125,0.15,0.175$, and 0.2 . Notice that these values of $\varepsilon$ are much smaller than the ones illustrated in Fig. 6 from the numerical stability analysis. However, these relatively small values of $\varepsilon$ are indeed large enough, as the following comparison of measurements and simulations verifies.

When each simulation reaches its steady state, FFT is used to calculate the amplitude of the torsional difference vibration (dof $12-\operatorname{dof} 2$ ) for the parametric excitation frequency $\omega_{P E}$. The results can be seen in Fig. 12. The grey lines represent measured amplitudes from Fig. 3, while the black lines originate from LTP-simulations as described above. Simulated and measured amplitudes match very well. Unsurprisingly for a linear system, there is a strictly linear relationship between maximum simulated amplitudes (dof $12-\operatorname{dof} 2$ ) of order 3.24 and parameter $\varepsilon$ (coefficient of determination: $R^{2}=99.97 \%$ ), which is not depicted here.

\section{SUMMARY AND CONCLUSION}

In this work, the parametric excitation of torsional vibrations in printing machines is analysed both experimentally and theoretically. First, various experimental data of these drivebelt-induced torsional vibrations are discussed. In experiment, unacceptably large vibration amplitudes occur during printing near a critical production speed, where the first drive belt order coincides with the machine's first eigenfrequency. Experiments at a test rig and at a sheet-fed offset printing machine indicate a very strong correlation of measured amplitudes. It is demonstrated experimentally, that active vibration control can

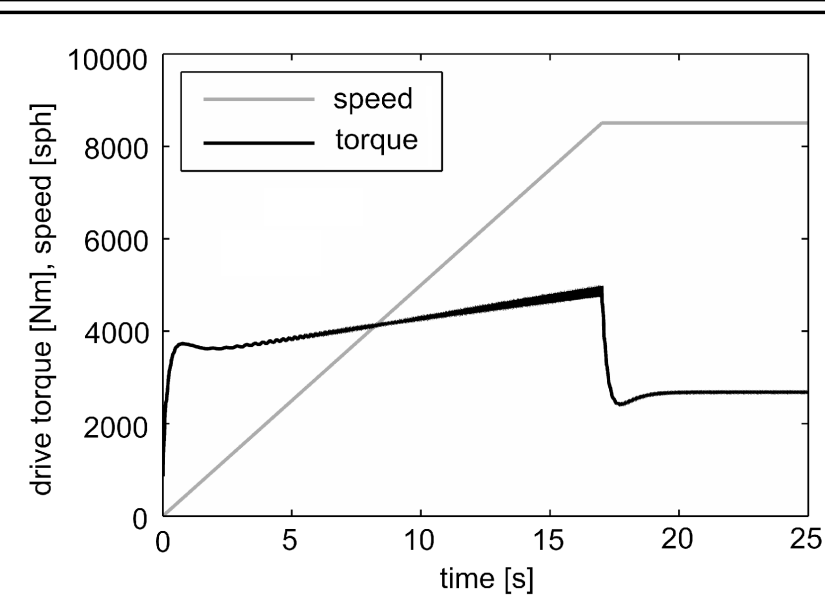

(a)

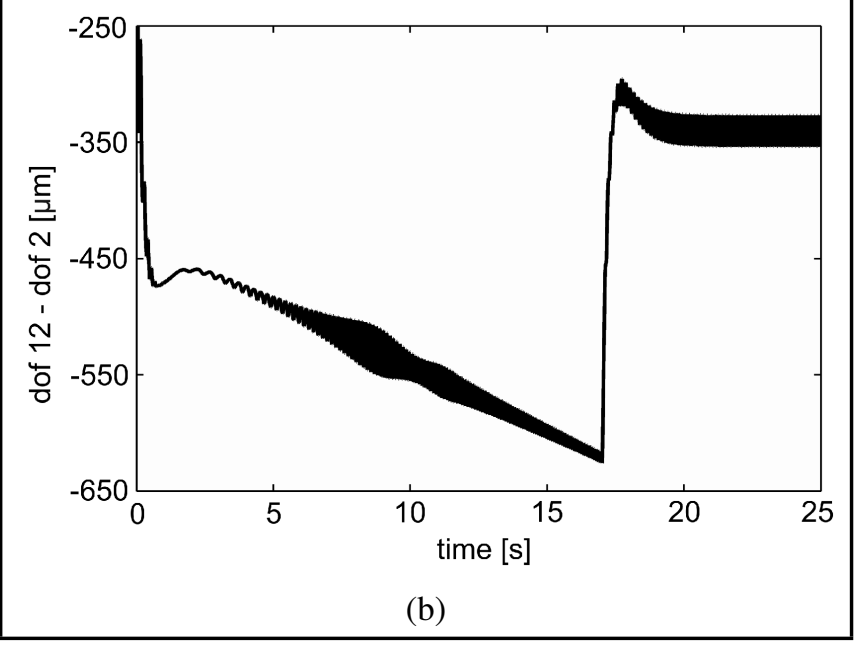

Figure 11. Results of LTP-Simulation: (a) desired speed (grey) and drive torque (black), (b) torsional difference vibration (dof $12-\operatorname{dof} 2$ ).

be used successfully to reduce these parametrically excited torsional vibrations in sheet-fed offset printing machines. In order to further explore this problem, the machine is modelled as a dynamical multi-degree of freedom system with time-periodic coefficients and a harmonic variation of the drive belt stiffness is studied. First, a numerical stability analysis is carried out employing Floquet theory. The drive control of the machine is modelled, and the system's damping parameters are determined from measurement data. This results in simulated vibration amplitudes that match very well with measured amplitudes. From these simulation results and from the numerical stability analysis presented in this work follows that the real machine does not become instable because the system's damping is too high and the parametric excitation parameter $\varepsilon$ is too small.

Future work could be aimed at comparing different machine types (plate cylinder diameters ranging from $0.180 \mathrm{~m}$ to $0.4854 \mathrm{~m}$, different belt pulley diameters, different belt profiles, different drive torques, etc.). In this work, experimental and numerical results for Heidelberg's largest sheet-fed offset printing machine were presented (plate cylinder diameter: $0.4854 \mathrm{~m})$.

Another objective of further research is the study of lateral vibrations of the drive belt by further measurements and a refined modelling. The motivation for the analysis of these 


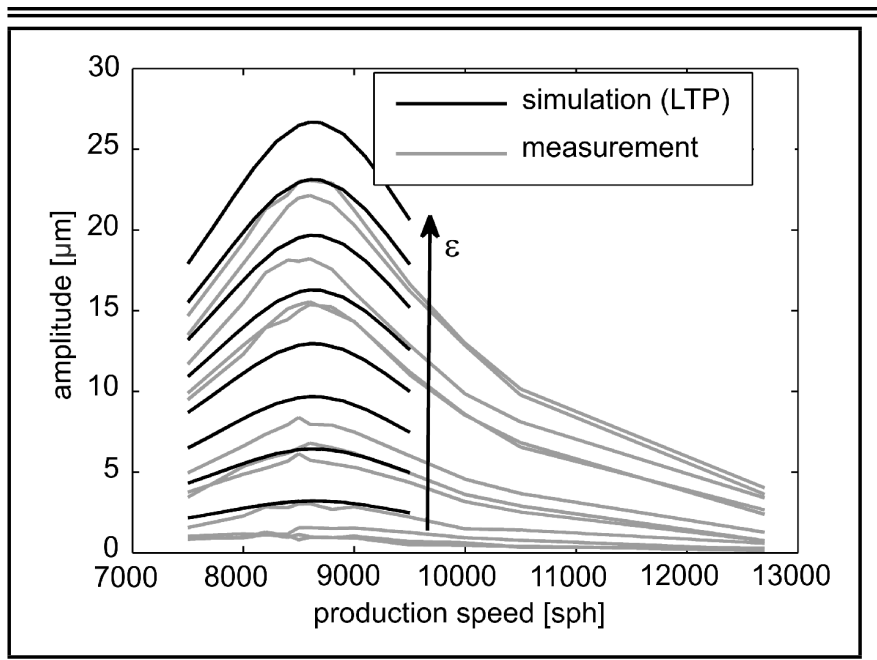

Figure 12. Simulated (LTP) and measured amplitudes of torsional difference vibration (dof 12 - dof 2) of parametric excitation frequency $\omega_{P E}$ (order 3.24).

lateral vibrations comes from work done by Dresig, who describes a drive belt with the coupled non-linear partial differential equations of longitudinal and lateral Euler-Bernoulli beam vibrations. ${ }^{10}$

\section{ACKNOWLEDGEMENTS}

This paper emerged as a result of the author's work for Heidelberger Druckmaschinen AG, for whom the author worked from January 2007 till June 2013 as a research engineer in Heidelberg, Germany. The author hereby expresses his deepest gratitude to his former colleagues and supervisors at Heidelberger Druckmaschinen AG who not only provided practical help and generously shared their insights, but who also encouraged the author to publish the results of this paper. Some findings reported in this paper were originally presented in German at the conference "8. VDI-Fachtagung Schwingungen in Antrieben 2013“ (November 5-6, 2013, Fulda, Germany), and the German conference paper was printed in the conference proceedings (ISBN: 978-3-18-092197-6, VDI-Berichte Nr. 2197). ${ }^{17}$

\section{REFERENCES}

1 Kipphan, H. (Ed.). Handbook of Print Media - Technologies and Production Methods, Springer-Verlag, Berlin, (2001). https://dx.doi.org/10.1007/978-3-540-29900-4

2 Kipphan, H. (Ed.). Handbuch der Printmedien - Technologien und Produktionsverfahren, Springer-Verlag, Berlin, (2000). https://dx.doi.org/10.1007/978-3-642-57024-7

3 Wiese, H. Der Beitrag antriebsdynamischer Untersuchungen im Entwicklungsprozeß von Bogenoffsetdruckmaschinen, Vortragssammelband VVD 2000 Verarbeitungsmaschinen und Verpackungstechnik, 66-79, Dresden, (2000).

4 Holzweißig, F. Dynamische Untersuchung an der Zylindergruppe einer Offsetmaschine, TH Dresden, Dresden, (1959).
5 Yamaguchi, M., Takasaki, K., Hirooka, H., Fujimoto, S., and Furukawa, S. Development of dynamic absorber builtin roll of high-speed and high-quality sheet-fed printing press, Mitsubishi Heavy Industries Technical Review, 34 (1), 20-24, (1997).

6 Buck, B. Schwingungen im Antriebsstrang von Bogenoffset-Druckmaschinen, Workshop Schwingungen in Verarbeitungsmaschinen, Höchberg, (2002).

7 Messer, M. H. E. Schwingungen in Bogenoffsetdruckmaschinen: Ergebnisse experimenteller und rechnerischer Drehschwingungsanalysen, Jahrbuch 2011 des Vereins Deutscher Druckingenieure e.V., 59-70 (2011).

8 Buck, B., Knopf, E., Schreiber, S., and Seidler, M. Nichtlineare Schwingungsphänomene in Bogenoffsetdruckmaschinen, VDI-Schwingungstagung, Leonberg, 345-361, (2005).

9 DIN 7867 Keilrippenriemen und -scheiben (V-ripped belts and corresponding pulleys), DIN Deutsches Institut für Normung e.V., Berlin, (1986).

10 Dresig, H. Schwingungen mechanischer Antriebssysteme: Modellbildung, Berechnung, Analyse, Synthese, SpringerVerlag, Berlin, (2001). https://dx.doi.org/10.1007/978-3662-09833-2

11 Sauer, B. Stationäre Schwingungen von Keilriementrieben im Frequenzbereich bis $240 \mathrm{~Hz}$, VDI-Verlag, Düsseldorf, (1988).

12 Bischoff, S. Schwingungsanregung von Druckmaschinen durch den Riementrieb, Heidelberger Druckmaschinen AG, internship report (22 pages), (2008).

13 Nöll, M. Kompensation diskreter Drehschwingungen bei Bogenoffsetdruckmaschinen, TU Darmstadt, Darmstadt, 63-79, (2004). http://tuprints.ulb. tu-darmstadt.de/503/1/Diss_Noell.pdf

14 Gross, D., Hauger, W., Schröder, J., Wall, W. A., and Govindjee, S. Engineering Mechanics 3, Dynamics, Springer-Verlag, Berlin, (2011). https://dx.doi.org/10.1007/978-3-642-14019-8

15 Rand, R. Lecture Notes on Nonlinear Vibrations, Department of Theoretical and Applied Mechanics, Cornell University, Ithaca, New York, (2005).

16 Dohnal, F. A contribution to the mitigation of transient vibrations: parametric antiresonance, theory, experiment and interpretation, TU Darmstadt, Darmstadt, (2012).

17 Messer, M. H. E. Parametererregte Drehschwingungen im Antriebsstrang von Bogenoffsetdruckmaschinen, 8. VDIFachtagung Schwingungen in Antrieben 2013 in Fulda, 1-12, VDI-Berichte Nr. 2197, Düsseldorf: VDI-Verlag, (2013). 